\title{
Subthreshold Nanosecond Laser, from Trials to Real-Life Clinical Practice: A Cohort Study
}

\author{
Hasan Chichan ${ }^{1,2}$ \\ Matthias Maus ${ }^{2, *}$ \\ Ludwig M Heindl ${ }^{1,3, *}$ \\ 'Department of Ophthalmology, \\ University of Cologne, Faculty of \\ Medicine and University Hospital \\ Cologne, Cologne, 50923, Germany; \\ ${ }^{2}$ Sehkraft Eye Center, Cologne, 50667. \\ Germany; ${ }^{3}$ Center for Integrated \\ Oncology (CIO) Aachen-Bonn-Cologne- \\ Duesseldorf, Cologne, 50937, Germany
}

*These authors contributed equally to this work
Correspondence: Hasan Chichan

Email chichan@sehkraft.de
Background: Age-related macular degeneration (AMD), a degenerative disorder, is a major cause of irreversible severe visual deterioration in the older Western population. Thus, treatments that resolve drusen may prove helpful in limiting the progression of AMD. The study aimed to evaluate the clinical outcome of sub-threshold nanosecond laser therapy (SNL) using a double-frequency nanosecond-pulsed laser with discontinuous energy beam distribution in the early stages of age-related macular degeneration (AMD).

Methods: In a single-center cohort study, 64 eyes with early or intermediate AMD were treated using SNL and then compared to 77 untreated, control eyes. The primary outcome measures included the area and amount of drusen, and the secondary outcome measures included change in visual acuity and rate of drusen progression within 6 months of follow-up.

Results: A total of 64 patients' eyes following SNL treatment showed a significant reduction in the area and amount of drusen after 6 months in comparison to the corresponding values in the control group $(p<0.001$, respectively). There was no significant difference in visual acuity change after 6 months in both groups ( $p \geq 0.59$, respectively). Within the 6-month followup, the rate of progression in drusen size and number was significantly lower in the SNL group $(26 \%)$ than in the control group $(69 \% ; p<0.001)$.

Discussion: SNL therapy is a novel, promising approach for improving the macular morphology in patients with early and intermediate AMD. Further studies are necessary to reveal the potential to reduce the risk of transformation into advanced or wet AMD and to retain vision. Keywords: subthreshold nanosecond laser therapy, age-related macular degeneration, ophthalmology, drusen, ocular surgery

\section{Introduction}

Age-related macular degeneration (AMD), a degenerative disorder, is a major cause of irreversible severe visual deterioration in the older Western population ${ }^{1 .}$ The epidemiological data reflects AMD progression more in the geriatric population, which makes it a serious global health issue because the elderly population is expected to double in the upcoming century. Global pervasiveness for late AMD is $0.37 \%$ and for early AMD is $8.01 \%$, with worldwide cumulative disease prevalence of $8.69 \%$ and $12.3 \%$, respectively, among the European population. Late AMD has two forms that can progress with time: atrophic AMD and neovascular AMD. ${ }^{2,3}$ It has already been shown that the type, size, and extent of drusen are considered to be predictors of AMD progression. ${ }^{4-6}$ Thus, treatments that resolve drusen may prove helpful in limiting the progression of AMD.

Early AMD does not cause any subjective vision complaints, and the progression to the later vision-threatening forms of the disease is almost always gradual, providing a good opportunity to use prophylactic treatment. Thermal laser 
photocoagulations with continuous wave $(\mathrm{CW})$ lasers have achieved only limited success due to the resulting thermal damage. ${ }^{7,8}$ The reported evidence of drusen reduction by using lasers and its correlation with AMD progression is still questionable, ranging from a positive impact $^{9}$ to no effect $^{10}$ or even increasing the risk of developing late AMD and the incidence of CNV, microscotomas, and subretinal fibrosis. ${ }^{11}$ The current reported evidence regarding thermal lasers suggests that the transition rate to more advanced stages of AMD was not decreased compared to untreated patients. ${ }^{12}$ Failure to stop AMD progression after using these thermal lasers could be due to retinal injury. ${ }^{8}$

A 3-nanosecond pulsed laser has the same properties as a continuous wave laser, but with a much shorter pulse duration. This tool decreases the destruction of retinal neurons caused by the heat generated through conventional 532-nm lasers. ${ }^{13,14}$ The advantage of using a nanosecond pulsed laser is the selective targeting of the retinal pigment epithelium (RPE). ${ }^{15}$ The reason for macular improvement after an injury to RPE is still vague; an improvement of the Bruch membrane conductive response could be due to excretion of MPPs (metalloproteinases) and RPE relocation. ${ }^{16}$ However, new blood vessels growth of the macula named as "choroidal neovascularisation CNV" is commonly observed as an AMD complication and patients already having one eye with $\mathrm{CNV}$ complaint are usually at higher risk of $\mathrm{CNV} .{ }^{12}$

As a result, macular morphological improvement occurs without retinal thermal damage, providing prophylactic use for early age-related macular degeneration (AMD). ${ }^{17} \mathrm{~A}$ major limitation in the current literature is the lack of data regarding the clinical development of symptoms and complications of early and intermediate AMD patients treated with subthreshold nanosecond laser (SNL). A better understanding of the safety and specific effects of nanosecond laser treatment on retinal, RPE, and choroidal tissues is needed in advising patients regarding prognosis, assessing new treatment options, and providing evidence-based interventions.

\section{Objective}

The objective of our study is to evaluate the clinical change in the area and number of drusen in participants with early and intermediate AMD treated with SNL and to assess the correlated change in visual acuity in the early stages of AMD within 6 months of follow-up.

\section{Methodology}

\section{Study Design}

This study was conducted in a single center in Cologne, Germany and was designed as a cohort study and performed in accordance with good clinical practice (International Conference on Harmonization of Technical Requirements of Pharmaceuticals for Human Use (ICH) E6) and the Declaration of Helsinki II. All patients were fully informed in detail about the therapy and the possible complications, and written informed consent was obtained from all patients before initiation of the treatment. According to national medical regulations for observational single-center studies, the Ethics Committee of the University of Cologne ruled that approval was not required for this study. All tenets of the Declaration of Helsinki and applicable national regulations and laws were observed.

\section{Participation and Eligibility}

The study cohorts were patients who were treated during the 2017-2018 period and were diagnosed with AMD. The data was extracted from the electronic written records of AMD patients and patients were examined and registered at the participating institution. To ensure that those cases were newly-diagnosed and to avoid the potential confounding factors of other conditions, we excluded subjects who were diagnosed with AMD in addition to other ocular conditions that could affect the outcomes of interest in the last 6-month period $(n=197)$. From the remaining 212 patients, we divided data into two groups, arm 1 received SNL treatment while arm 2 is the control group (Figure 1).

Subjects were not enrolled into the study if they exhibited retinal thickening or had undergone any ocular surgery. All participants underwent a standardized ophthalmic examination, slit-lamp examination of the anterior and posterior segments, color fundus photography, auto-fluorescence images, infrared images, and optical coherence tomography (OCT) scans of the macula (Topcon, Optical 3D-Coherence Topography Dri OCT Triton Plus) were performed pretreatment and after 6 months of follow-up and anamnesis of the past medical and ocular history. A fluorescein angiography was performed when a wet AMD was suspected.

The primary outcome measures included the area and number of drusen, and the secondary outcome measures included change in visual acuity and rate of drusen progression within 6 months of follow-up. Worse visual acuity was used to assess elementary lesions and cases were categorized into two types; geographic atrophy (GA) and choroidal new vessels $(\mathrm{CNV})$. 


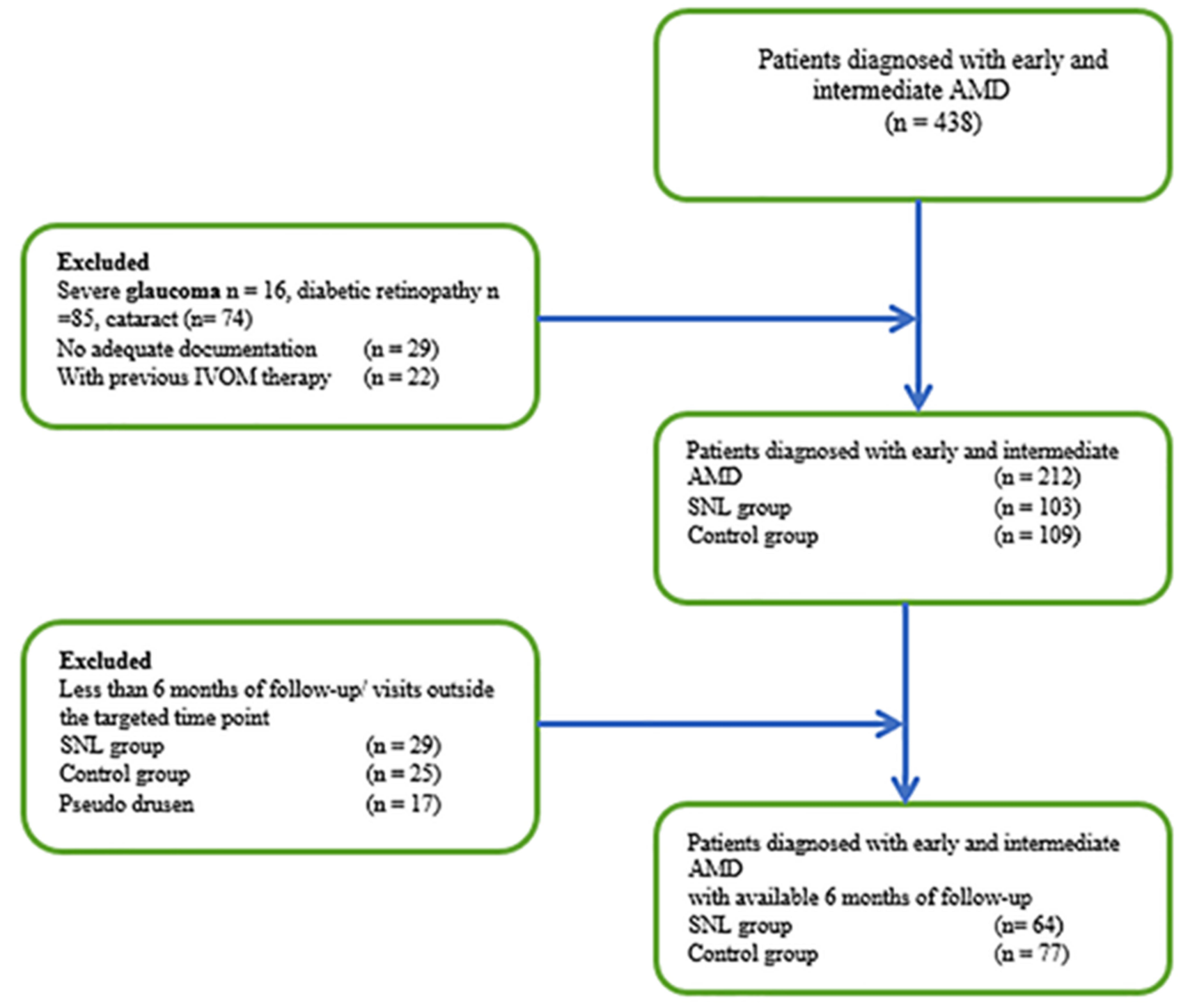

Figure I Patient inclusion and exclusion in two arms (SNL and control groups).

- Geographic atrophy "GA" presented as decreased coloration sharp demarcated zones with underlying large choroidal vessels exposure as appeared on ophthalmoscopy. These areas owned to window defect and were hyperfluorescent on fluorescein angiography "FA", and defined as "AMD natural end result in clinical evidence absence of choroidal neovascularization". Hence adjacent to disciform scar areas of retinal pigment epithelium "RPE" were not categorized as GA but instead RPE depigmentation.

- CNV was categorized as vascularized RPE detachment, occult, and well CNV, respectively. Well-defined CNV was categorized by well-defined boundaries and bright, early hyper-fluorescence of choroidal. These boundaries become concealed with progressive dye transit through widespread and speedy dye leakage in sub-sensory retina. On early angiography phase occult CNV did not display discernible hyper-fluorescence. However, late and mid phase frames showed mild and diffused leakage into sub-sensory retina and outer retina displayed stippled hyper-fluorescence. Vascularized RPE detachments were presented by red free and color photographs and uneven and delayed filling during FA course.

The SNL laser (Ellex, Adelaide, Australia) has a 3-nanosecond pulse duration at the $532 \mathrm{~nm}$ wavelength. The laser spot size is set at a $400 \mu \mathrm{m}$ diameter, with an energy distribution beam profile applied coaxially by using a slit-lamp microscope, a digital interface to record the number of pulses, 
energy/pulse, and a contact lens (Area Centralis Volk Ophthalmic, Inc.). Each patient received, in a single session, 24-26 spots that were targeted between the superior and inferior vessels of the macula. The energy was modified according to the individual findings depending on corneal, lens, or vitreous opacities and retinal pigmentation variations. Participants received 24-26 spots, titrated to suit each participant, around the macula with an average $0.24 \mathrm{~mJ}$ laser energy at every treatment spot. The spots should be titrated (generally 2-3 trials) to be under the visual threshold for retinal change (range $=0.15-0.45 \mathrm{~mJ}$ ) with a $0.19 \mathrm{~J} / \mathrm{cm}^{2}$ mean radiant exposure $(0.12-0.36$ range).

\section{Quantification Parameters and Distance Calibration}

Drusen size and number was determined by examining fundus photographs (color fundus photography, monochromatic red-free fundus photography) at baseline and 6 months for analysis using ImageJ (freeware Java version; National Institutes of Health, Bethesda, MD). The baseline fundus image and the follow-up image were allied using the TurboReg plug-in (Biomedical Imaging Group; Swiss Federal Institute of Technology, Lausanne, Switzerland) for ImageJ. The spatial calibration was performed to gather measurements from the images using a real spatial unit $(\mu \mathrm{m})$. By using a stage micrometer image, a conversion from pixels to micrometers was performed while using the same magnification and resolution. Then, an automatic counting was performed if the image did not have too many individual drusen touching each other. Otherwise, manual particle counting was performed using the multi-point and area selection tools.

The automatic particle analysis required "binary" black and white image. These images were converted to a binary image 8-bit. The overlapping objects in a binary image were separated using watershed separation. A threshold range was set to tell the objects of interest apart from the background. All image pixel values under the threshold were converted to black, and all pixels with values above the threshold were converted to white. The accuracy of image registration was individually confirmed and compared at baseline and 6 months within ImageJ, and then all images were examined for artefacts before registration. The proportions of laser-treated eyes with a reduced drusen area were compared with those observed in the natural history control group (Figure 2).
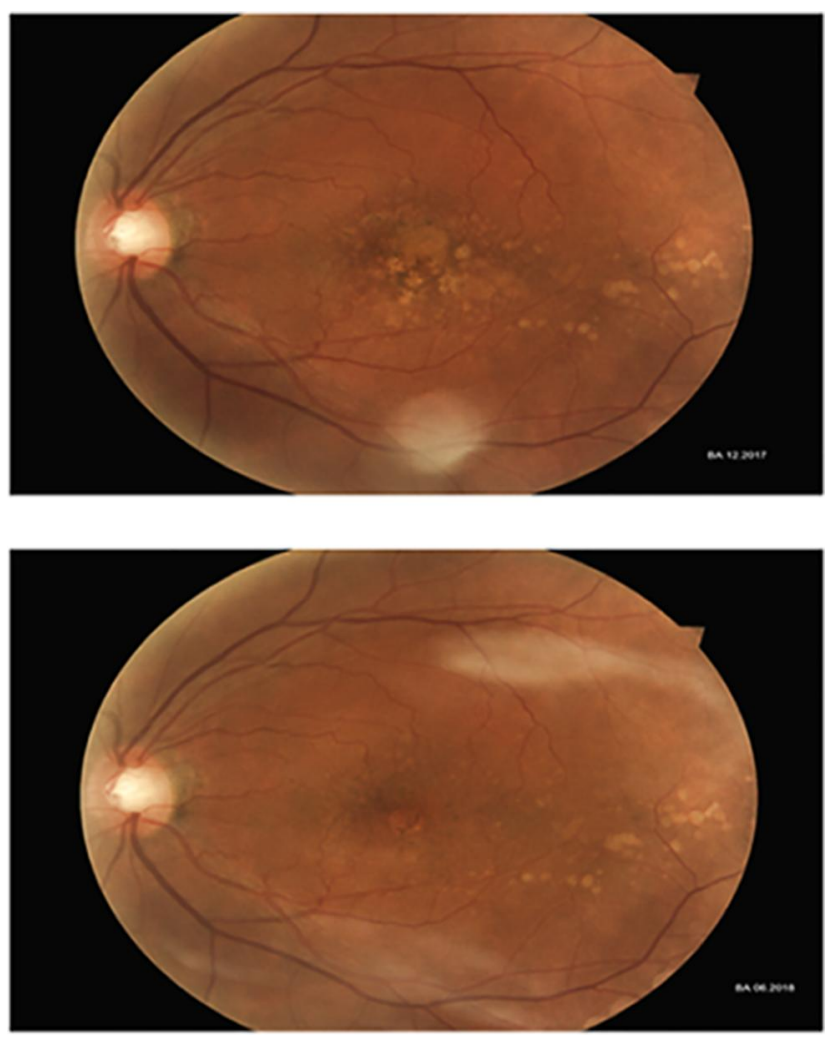

Figure 2 Fundus photograph showing the macular morphological features 6 months after using SNL therapy.

Pseudodrusen were identified and excluded from the study. ${ }^{5,18}$ All eyes were examined to check for the following signs of progression: conversion from small $(<63 \mu \mathrm{m})$ to intermediate $(63-125 \mathrm{~m})$ or large drusen $(<125 \mu \mathrm{m})$, geographic atrophy and choroidal neovascularization., 4 Any sharp hypopigmentation of the RPE was considered geographic atrophy when it was larger than $175 \mu \mathrm{m} .^{20}$ Retinal pigment changes were not discarded since the laser treatment itself could lead to pigment abnormalities in the area treated.

\section{Statistical Analysis and Data Collection}

Data was collected on Microsoft Office Excel 2010, whereas statistical analysis was performed with SPSS 19.0 software. VAs were recorded and converted from Snellen to Log MAR for statistical analysis. ${ }^{21}$ The results were checked for normal distribution using a histogram and Shapiro-Wilk test.

\section{Results}

Two hundred and twelve patients were screened. We divided data into two groups, arm 1 received SNL treatment while arm 2 was the control group. Median age for 
AMD enrolment was 65 (45-80) years. Ninety-six of the participants were male and 116 participants were female. The range for BCVA was between $60(6 / 18)$ early treatment diabetic retinopathy study letters to $93(6 / 4.8)$. Baseline images were collected for all enrolled participants and follow-up results were evaluated over the period of 6 months. Environmental and general risk factors evaluation presented $37 \%(\mathrm{n}=79)$ of patients on medication treatment with anti-hypertensive, $31 \%(\mathrm{n}=65)$ has a history of angina or other heart diseases and $32 \%(n=68)$ of participants had a history of smoking (Table 1).

In the control group the average drusen number at baseline was $37.3 \pm 21.1$ (range $=6-88$ ) which was significantly $(p<0.001,95 \% \mathrm{CI}=36.9-50.3)$ increased after 6 months follow-up with a mean value of 43.6 \pm 24.5 (range=9-90). Drusen number at baseline was $29.4 \pm 19.2$ (range $=3-74$ ), and after 6 months, the drusen number was reduced to $24.7 \pm 14.8$ (range $=1-29$ ) in the SNL arm. Significant reduction $(p<0.001,95 \% \mathrm{CI}=20.6-28.7)$ in the $\mathrm{SNL}$ group was observed after a period of 6 months (Figure 3).

The average drusen size $(\mu \mathrm{m})$ in the control group at baseline was 40.6 \pm 20.3 (range=12.4-93.1) and after 6 months the average size was increased to $49.3 \pm 28.4$ (range $=11-116.5$ ), which was statistically significant $(p<0.001,95 \% \mathrm{CI}=39.4-59.2)$. In the SNL arm, the drusen size $(\mu \mathrm{m})$ at baseline was $59.9 \pm 44.8$ (range $=7.1-171)$, and after 6 months, $48.9 \pm 34.4$ (range $=4.8-125.8$ ), revealing a significant reduction $(p<0.001,95 \% \mathrm{CI}=36.9-61$, Figure 4$)$. The best corrected visual acuity (logMAR) in the control group was $0.2 \pm 0.21$ (range $=0.0-0.7$ ) at baseline and $0.2 \pm 0.19$ (range $=0.0-0.7)$ after 6 months with no statistical difference $(p=0.59,95 \% \mathrm{CI}=0.1-0.3)$. The best

Table I Representation of Baseline Characteristics for Patients Enrolled (SNL and Control Group)

\begin{tabular}{|l|c|}
\hline \multicolumn{2}{|l|}{ Characteristics } \\
\hline Median Age (Years) & 65 \\
\hline $\begin{array}{l}\text { Gender } \\
\text { Male }\end{array}$ & $\mathbf{N}(\%)$ \\
Female & $96(45 \%)$ \\
\hline Environmental and Systemic Factors & $16(55 \%)$ \\
Anti-Hypertensive Medications & $79(37 \%)$ \\
Cardiovascular Event History & $65(31 \%)$ \\
\hline Smoking History & \\
Smokers & $68(32 \%)$ \\
Non Smokers & $144(68 \%)$ \\
\hline
\end{tabular}

corrected visual acuity ( $\log \mathrm{MAR})$ in the SNL group was $0.18 \pm 0.12$ (range $=0.0-4.0$ ) at baseline and $0.15 \pm 0.12$ (range $=0.0-0.4$ ) after 6 months with no statistical significance $(p=0.63,95 \% \mathrm{CI}=0.1-0.2)$, shown in Figure 4 . Within the 6-month follow-up, the rate of drusen progression was $26 \%$ in the SNL group and $69 \%$ in the control group with a significant difference $(p<0.001)$. None of the patients showed any severe complications, such as uveitis, retinal detachment, retinal bleeding, or vision loss.

A total of 198 patients were classified as GA and 111 (56\%) of these were female. Drusen association with GA was observed in $167(84 \%)$ of the cases, and $31(16 \%)$ patients showed an association with RPE, however hypopigmentation was a rare observation in six (3\%) of the cases. Average patient age for GA was 65 years. Similarly, for exudative AMD, 202 (95\%) eyes were reported with a median age 65 years. Occult CNV was harbored in most participants eyes, 149 (73\%), well defined CNV in 40 (20\%), and vascularised detachment in $13(6 \%)$ of the cases (Table 2).

\section{Discussion}

The current study was conducted to evaluate the functional and morphological effects of SNL with respect to its safety and efficacy. Moreover, it also followed the patients retrospectively to observe the clinical development, related complications, and the outcome of early and intermediate AMD patients treated with SNL and compared the results with an untreated group. Other significant markers of AMD have been identified in serum and urine, nevertheless drusen is considered as a classical marker. It is observed that SNL therapy appeared to be effective in improving macular morphology by reducing markers of AMD progression. ${ }^{22,23}$

In previous thermal laser studies, serious ocular adverse events have been reported. These include retinal hemorrhage, deterioration of vision from baseline (two or more logMAR lines), RPE detachments, RPE atrophy, or cataracts. $^{12,24,25}$ Contrary to these findings, we performed BCVA, fundoscopy, and OCT examinations to identify potential complications. None of the patients showed severe complications. OCT examination significance was also established in study by Alamouti and Funk ${ }^{26}$ evaluating total retinal thickness scanning through OCT, resulting in a high reproducibility " $3.5 \%$ coefficient of variance". A clinical trial ${ }^{27}$ reported no significant delay with SNL treatment in late AMD progress in the non-study eye $(p=0.611)$ and the decline in BCVA rate was higher in 


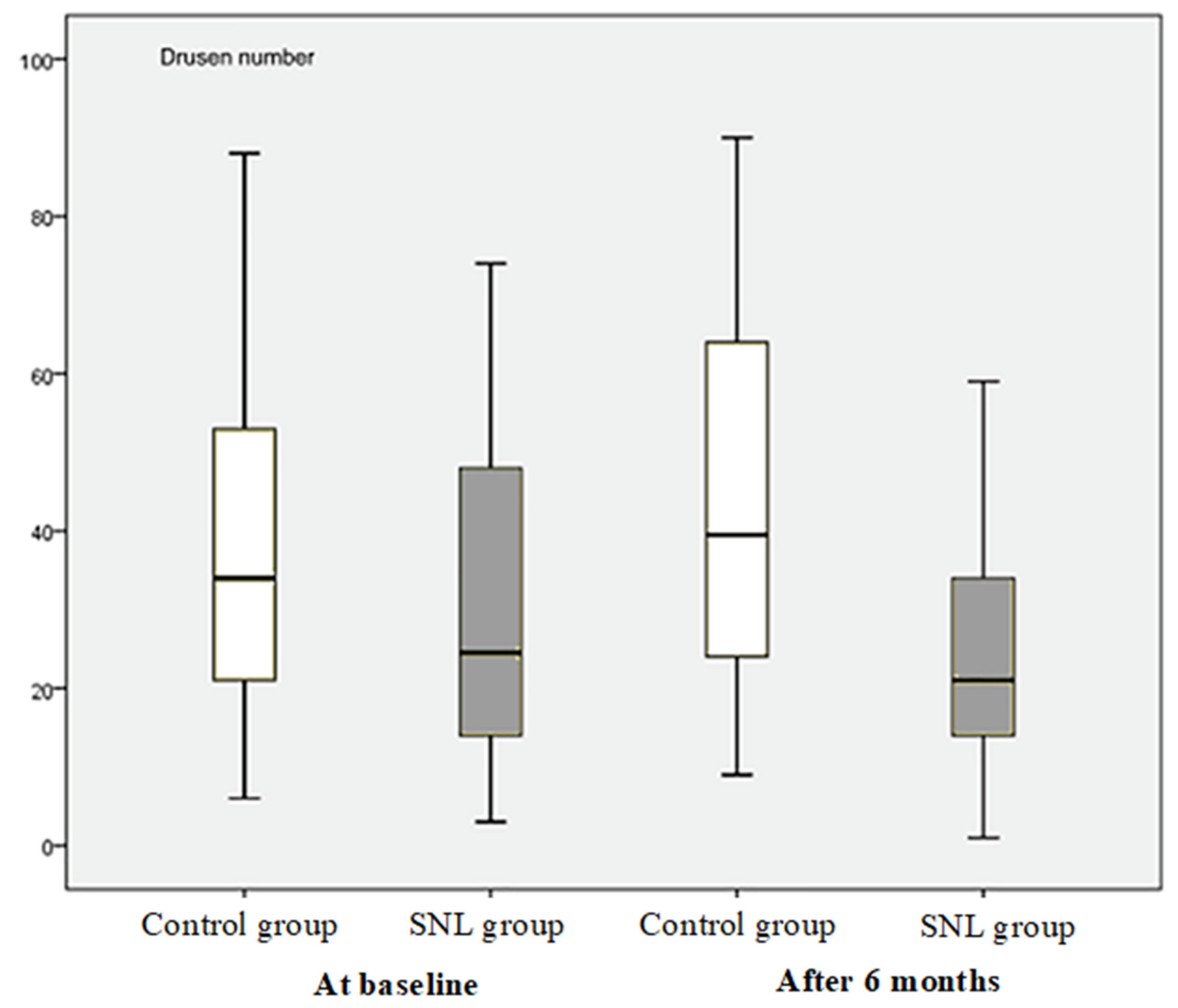

Figure 3 The drusen number in the control group at baseline was 37.3 \pm 21.1 (range=82.6-88), and after 6 months follow-up, the number was $43.6 \pm 24.5$ (range=81.9-90, $p<0.001,95 \% \mathrm{Cl}=36.9-50.3$ ). In the SNL group, the drusen number at baseline was $29.4 \pm 19.2$ (range=7I.3-74), and after 6 months it was $24.7 \pm \mid 4.8$ (range $=58.1-59$, $p<0.001,95 \% \mathrm{Cl}=20.6-28.7)$.

the SNL group participants in comparison to the sham treatment group in the study eye $(p<0.001)$ but found a non-significant correlation in the non-study eye $(p=0.628)$, concluding that one eye SNL did not delay late AMD progression in the fellow eye.

The 3-ns pulsed laser has similar features to those of conventional 532-nm continuous wave (CW) photocoagulation lasers; however, the pulse duration selectively modulates pigmented tissues while minimizing thermal damage to the delicate apposing retinal neurons. ${ }^{28}$ Thermal laser photocoagulation with $\mathrm{CW}$ lasers was used, and it was reported to reduce the drusen load. However, it failed to show promising results because of the thermal damage resulting from the $\mathrm{CW}$ laser causing injury to the photoreceptors and inner retinal neurons, since the energy of that laser is transformed to heat energy through absorption by the melanin in the RPE monolayer and choroid. ${ }^{2}$ Using very short laser pulses ( $3 \mathrm{~ns}$ ), an insult caused by steam production around melanosomes can be confined to the retinal pigment epithelium (RPE), inducing a highly selective and discrete non-thermal injury. ${ }^{7}$ While the mechanism by which the insult to RPE cells brings about a beneficial change in the macula is not well understood, it has been hypothesized that a $3 \mathrm{~ns}$ laser could induce the migration of RPE cells and release of matrix metalloproteinases, improving the hydraulic conductivity of Bruch's membrane. ${ }^{16}$ Irrespective of the functional mechanism, it is observable that several beneficial effects on the macula occurred with traditional thermal lasers without major complications.

Few complications were observed in our study that were associated with SNL therapy and this might be due to the complexity of cases. The extent of the initial retinal changes and the deposition of extra-cellular deposits between the RPE and Bruch's membrane and the damage to the photoreceptors could be significantly associated with the effectiveness of the treatment. Our findings were compatible with previous studies in which drusen regress without a focal vision loss or new vessel growth in the retina following prophylactic laser treatment. ${ }^{17,28}$ Nonetheless, a study by Rosenfeld and Feuer ${ }^{29}$ explained that SNL treatment during intermediate AMD is not that beneficial with a risk of vision loss and accelerated disease progression with RPD. However, another LEAD study has shown that SNL 


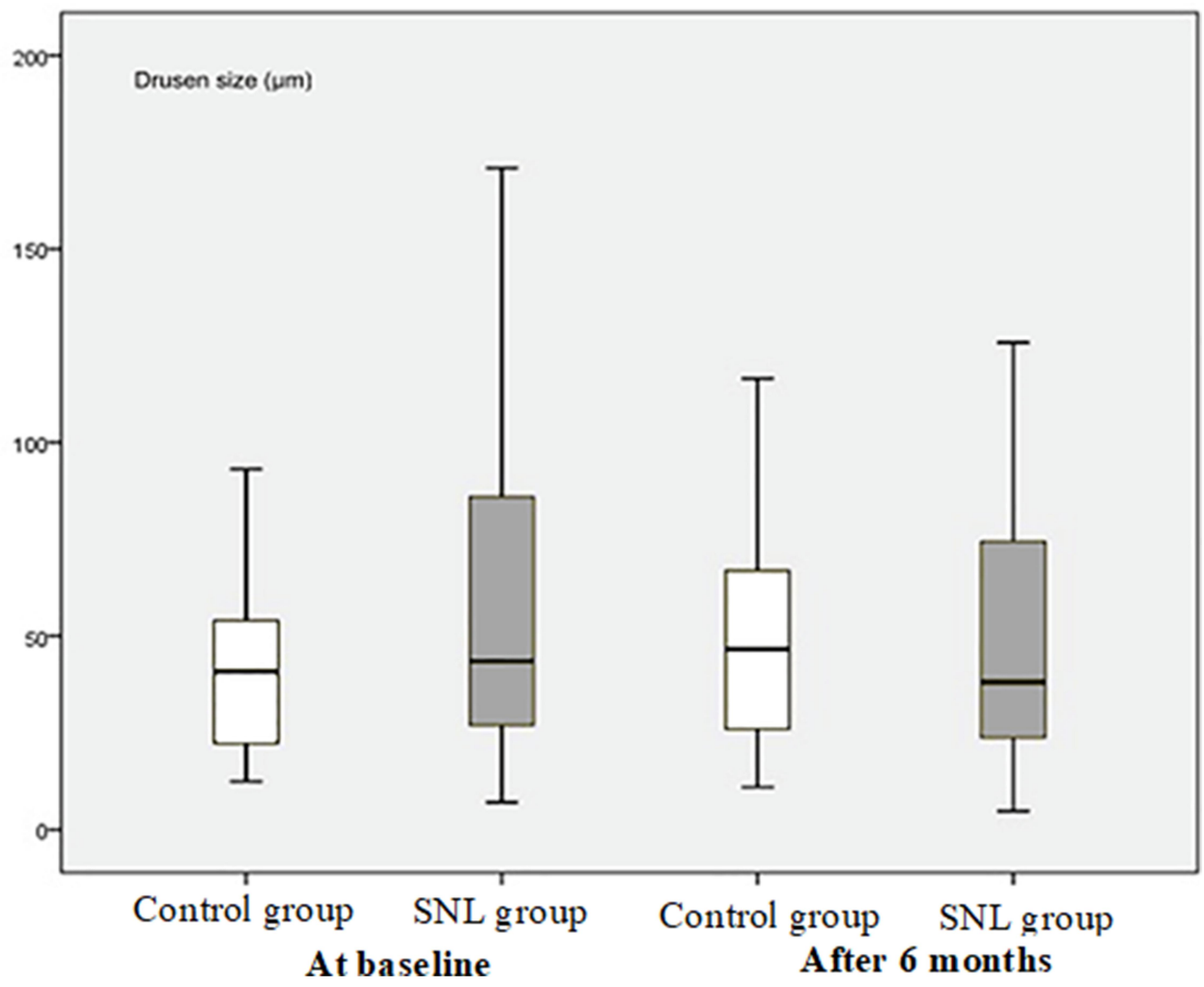

Figure 4 The drusen size $(\mu \mathrm{m})$ in the control group at baseline was $40.6 \pm 20.3$ (range=80.7, $12.4-93.1$ ), after 6 months it was $49.3 \pm 28.4$ ( range $=105.5$, II-II6.5, $p<0.001$, $95 \% \mathrm{Cl}=39.4-59.2)$. In the SNL group, the drusen size $(\mu \mathrm{m})$ at baseline was $59.9 \pm 44.8($ range $=|63.9,7.1-17|)$, and after 6 months it was $48.9 \pm 34.4($ range $=12 \mid$, 4.8-125.8, $p<0.00 \mathrm{I}, 95 \% \mathrm{Cl}=36.9-6 \mathrm{I})$.

treatment might be associated with slow progression without coexistent RPD. ${ }^{16}$ The coexistence of RPD has been a newlyrecognized form of late $\mathrm{AMD} .^{6,18}$ One can postulate that the macular morphology could be improved if prompt treatment is

Table 2 Measurement of Secondary Outcome Variables Geographic Atrophy and Choroidal New Vessels

\begin{tabular}{|l|c|}
\hline \multicolumn{2}{|l|}{ Secondary Outcome Measures } \\
\hline Geographic Atrophy (GA) & \\
Total GA patients & 198 \\
Female GA patients & $111(56 \%)$ \\
Male GA patients & $87(44 \%)$ \\
Drusen Association with GA & $167(84 \%)$ \\
RPE Association with GA & $31(16 \%)$ \\
Hypo-pigmentation & $6(3 \%)$ \\
\hline Choroidal New Vessels (CNV) & \\
Total CNV Patients & $202(95 \%)$ \\
Median age & 65 \\
Occult CNV & $149(73 \%)$ \\
Well-Defined CNV & $40(20 \%)$ \\
Vascularized Detachment & $13(6 \%)$ \\
\hline
\end{tabular}

induced after diagnosis of the earlier signs of AMD. Therefore, a practical option would be early intervention. This may help to maintain a good macular morphological appearance as well as reduce the challenges and costs of progression to late-stage AMD. This could be achieved through a simple routine eye check before the occurrence of devastating complications. The current use of nutritional supplements for early AMD has achieved only limited success. $^{4,30}$ This raises the need for a novel and innovative approach that could reverse the early clinical indicators of AMD. A detailed evaluation of the clinical impact and the possible longterm adverse events of SNL therapy in large, prospective, controlled clinical studies is needed. Although previous investigations have shown no evidence of any serious adverse effects related to the therapy in the energy used under the visual threshold for retinal change, further studies are required to reveal the significance of SNL therapy to reduce the risk of transformation into advanced or wet AMD.

There were few limitations that should be considered; first the small sample size was studied. A limited numbers of patients were eligible as per the study inclusion criteria. Confounders such as lens opacities and retinal changes have been identified to have an impact on the outcome. As it was 
a retrospective cohort study, selection bias and non-matching of controls may be considered as a limitation of the study.

\section{Conclusion}

SNL therapy is a novel, promising approach for improving the macular morphology in patients with early and intermediate AMD. Further studies are necessary to reveal the potential to reduce the risk of transformation into advanced or wet AMD and to retain vision.

\section{Data Sharing Statement}

The datasets generated during and/or analyzed during the current study are not publicly available due to the DSGVO medical data regulations in Germany but are available from the corresponding author on reasonable request.

\section{Acknowledgment}

Matthias Maus and Ludwig M. Heindl both should be considered as equal senior authors.

\section{Author Contributions}

All authors made substantial contributions to the conception and design, acquisition of data, or analysis and interpretation of data; took part in drafting the article or revising it critically for important intellectual content; agreed to submit to the current journal; gave final approval of the version to be published; and agree to be accountable for all aspects of the work.

\section{Disclosure}

We declare that the authors have no competing interests as defined by Nature Research or other interests that might be perceived to influence the results and/or discussion reported in this paper. The corresponding author is responsible for submitting a competing interest's statement on behalf of all authors of the paper.

\section{References}

1. Birch DG, Liang FQ. Age-related macular degeneration: a target for nanotechnology derived medicines. Int J Nanomedicine. 2007;2(1):65. doi:10.2147/nano.2007.2.1.65

2. Christensen K, Doblhammer G, Rau R, Vaupel JW. Ageing populations: the challenges ahead. Lancet. 2009;374(9696):1196-1208. doi:10.1016/S0140-6736(09)61460-4

3. Chirco KR, Sohn EH, Stone EM, Tucker BA, Mullins RF. Structural and molecular changes in the aging choroid: implications for age-related macular degeneration. Eye. 2017;31(1):10-25. doi:10.1038/eye.2016.216

4. Ferris FL, Davis MD, Clemons TE, et al. A simplified severity scale for age-related macular degeneration: AREDS Report No. 18. Arch Ophthalmol. 2005;123(11):1570-1574.
5. Zhou Q, Daniel E, Maguire MG, et al.; Comparison of Age-Related Macular Degeneration Treatments Trials Research Group. Pseudodrusen and incidence of late age-related macular degeneration in fellow eyes in the comparison of age-related macular degeneration treatments trials. Ophthalmology. 2016;123(7):1530-1540. doi:10.1016/j.ophtha.2016.02.043

6. Gil JQ, Marques JP, Hogg R, et al. Clinical features and long-term progression of reticular pseudodrusen in age-related macular degeneration: findings from a multicenter cohort. Eye. 2017;31(3):364-371. doi:10.1038/eye.2016.207

7. Wood JP, Plunkett M, Casson RJ, Chidlow G. Retinal damage profiles and neuronal effects of laser treatment: comparison of a conventional photocoagulator and a novel 3-nanosecond pulse laser. Invest Ophthalmol Vis Sci. 2013;54(3):2305-2318. doi:10.1167/iovs.12-11203

8. Mojana F, Brar M, Cheng L, Bartsch DU, Freeman WR. Long-term SD-OCT/SLO imaging of neuroretina and retinal pigment epithelium after sub-threshold infrared laser treatment of drusen. Retina. 2011;31 (2):235. doi:10.1097/IAE.0b013e3181ec80ad

9. Ivandic BT, Ivandic T. Low-level laser therapy improves vision in patients with age-related macular degeneration. Photomed Laser Surg. 2008;26(3):241-245. doi:10.1089/pho.2007.2132

10. Figueroa M, Schocket LS, DuPont J, Metelitsina TI, Grunwald JE. Effect of laser treatment for dry age related macular degeneration on foveolar choroidal haemodynamics. Br J Ophthalmol. 2004;88 (6):792-795. doi:10.1136/bjo.2003.033837

11. Olk RJ, Friberg TR, Stickney KL, et al. Therapeutic benefits of infrared (810-nm) diode laser macular grid photocoagulation in prophylactic treatment of nonexudative age-related macular degeneration: two-year results of a randomized pilot study. Ophthalmology. 1999;106(11):2082-2090. doi:10.1016/S0161-6420(99)90487-6

12. Virgili G, Michelessi M, Parodi MB, Bacherini D, Evans JR. Laser treatment of drusen to prevent progression to advanced age-related macular degeneration. Cochrane Database Syst Rev. 2015;10.

13. Guymer RH, Brassington KH, Dimitrov P, et al. Nanosecond-laser application in intermediate AMD: 12-month results of fundus appearance and macular function. Clin Experiment Ophthalmol. 2014;42 (5):466-479. doi:10.1111/ceo.12247

14. Zhang JJ, Sun Y, Hussain AA, Marshall J. Laser-mediated activation of human retinal pigment epithelial cells and concomitant release of matrix metalloproteinases. Invest Ophthalmol Vis Sci. 2012;53 (6):2928-2937. doi:10.1167/iovs.11-8585

15. Wood JP, Plunkett M, Previn V, Chidlow G, Casson RJ. Nanosecond pulse lasers for retinal applications. Lasers Surg Med. 2011;43 (6):499-510. doi:10.1002/1sm.21087

16. Guymer RH, Wu Z, Hodgson LA, et al. Subthreshold nanosecond laser intervention in age-related macular degeneration: the LEAD randomized controlled clinical trial. Ophthalmology. 2019;126 (6):829-838. doi:10.1016/j.ophtha.2018.09.015

17. Jobling AI, Guymer RH, Vessey KA, et al. Nanosecond laser therapy reverses pathologic and molecular changes in age-related macular degeneration without retinal damage. FASEB J. 2015;29 (2):696-710. doi:10.1096/fj.14-262444

18. Spaide RF, Ooto S, Curcio CA. Subretinal drusenoid deposits AKA pseudodrusen. Surv Ophthalmol. 2018;63(6):782-815.

19. Peng Y, Dharssi S, Chen Q, et al. DeepSeeNet: a deep learning model for automated classification of patient-based age-related macular degeneration severity from color fundus photographs. Ophthalmology. 2019;126 (4):565-575. doi:10.1016/j.ophtha.2018.11.015

20. Dimitrov PN, Robman LD, Varsamidis M, et al. Relationship between clinical macular changes and retinal function in age-related macular degeneration. Invest Ophthalmol Vis Sci. 2012;53 (9):5213-5220. doi:10.1167/iovs.11-8958

21. Gregori NZ, Feuer W, Rosenfeld PJ. Novel method for analyzing snellen visual acuity measurements. Retina. 2010;30(7):1046-1050. doi:10.1097/IAE.0b013e3181d87e04 
22. Garrity ST, Sarraf D, Freund KB, Sadda SR. Multimodal imaging of nonneovascular age-related macular degeneration. Invest Ophthalmol Vis Sci. 2018;59(4):AMD48-64. doi:10.1167/iovs.18-24158

23. Guymer RH, Tao LW, Goh JK, et al. Identification of urinary biomarkers for age-related macular degeneration. Invest Ophthalmol Vis Sci. 2011;52(7):4639-4644. doi:10.1167/iovs.10-7120

24. Arnold JJ, Heriot W. Age related macular degeneration. BMJ Clin Evid. 2007;2007.

25. Choroidal Neovascularization Prevention Trial Research Group. Choroidal neovascularization in the choroidal neovascularization prevention trial. Ophthalmology. 1998;105:1364-1372. doi:10.1016/ S0161-6420(98)98014-9

26. Alamouti B, Funk J. Retinal thickness decreases with age: an OCT study. Br J Ophthalmol. 2003;87(7):899-901. doi:10.1136/bjo.87.7.899

27. Wu Z, Luu CD, Hodgson LA, et al. Secondary and exploratory outcomes of the subthreshold nanosecond laser intervention randomized trial in age-related macular degeneration: a LEAD study report. Ophthalmol Retina. 2019;3(12):1026-1034. doi:10.1016/j.oret.2019.07.008
28. Vessey KA, Ho T, Jobling AI, et al. Nanosecond laser treatment for age-related macular degeneration does not induce focal vision loss or new vessel growth in the retina. Invest Ophthalmol Vis Sci. 2018;59 (2):731-745. doi:10.1167/iovs.17-23098

29. Rosenfeld PJ, Feuer WJ. Warning: do not treat intermediate AMD with laser therapy. Ophthalmology. 2019;126(6):839-840. doi:10.1016/j.ophtha.2018.12.016

30. Krishnadev N, Meleth AD, Chew EY. Nutritional supplements for age-related macular degeneration. Curr Opin Ophthalmol. 2010;21 (3):184. doi:10.1097/ICU.0b013e32833866ee
Clinical Ophthalmology

\section{Publish your work in this journal}

Clinical Ophthalmology is an international, peer-reviewed journal covering all subspecialties within ophthalmology. Key topics include: Optometry; Visual science; Pharmacology and drug therapy in eye diseases; Basic Sciences; Primary and Secondary eye care; Patient Safety and Quality of Care Improvements. This journal is indexed on PubMed

Submit your manuscript here: https://www.dovepress.com/clinical-ophthalmology-journal
Dovepress

Central and CAS, and is the official journal of The Society of Clinical Ophthalmology (SCO). The manuscript management system is completely online and includes a very quick and fair peer-review system, which is all easy to use. Visit http://www.dovepress.com/ testimonials.php to read real quotes from published authors. 